\title{
Effects of the Endophytic Bacteria Bacillus cereus BCM2 on Tomato Root Exudates and Meloidogyne incognita Infection
}

\author{
Xia Li, ${ }^{1}$ Hai-Jing Hu, ${ }^{1,2}$ Jing-Yu Li, ${ }^{1}$ Cong Wang, ${ }^{1}$ Shuang-Lin Chen, ${ }^{1}$ and Shu-Zhen Yan ${ }^{1, \dagger}$ \\ ${ }^{1}$ Jiangsu Key Laboratory for Microbes and Functional Genomics, Jiangsu Engineering and Technology Research Center for In- \\ dustrialization of Microbial Resources, College of Life Sciences, Nanjing Normal University, Nanjing, Jiangsu Province \\ 210023, China \\ ${ }^{2}$ School of Life Science, Nanjing University, Nanjing, China
}

\begin{abstract}
Root-knot nematodes (Meloidogyne spp.) cause serious crop losses worldwide. The colonization of tomato roots by endophytic bacteria $\mathrm{Ba}$ cillus cereus $\mathrm{BCM} 2$ can greatly reduce Meloidogyne incognita damage, and tomato roots carrying BCM2 were repellent to $M$. incognita secondstage juveniles (J2). Here, the effects of BCM2 colonization on the composition of tomato root exudates was evaluated and potential mechanisms for BCM2-mediated $M$. incognita control explored using a linked twinpot assay and GC-MS. On water agar plates, J2 preferentially avoided filter paper treated with tomato root exudates (organic phase only) from plants inoculated with BCM2, visiting these $67.1 \%$ less than controls. In a linked twin-pot assay, BCM2 treatment resulted in a $42.0 \%$ reduction in the number of nematodes in the soil, a $43.3 \%$ reduction in the number of galls and a $47.7 \%$ decrease in the density of $M$. incognita in root

tissues. Analysis of root exudate composition revealed that BCM2 inoculation increased the number of components in exudates. Among these, 2,4-di-tert-butylphenol, 3,3-dimethyloctane, and n-tridecane secretions markedly increased. In repellency trials on water agar plates, J2 avoided 2,4-di-tert-butylphenol, n-tridecane, and 3,3-dimethyloctane at concentrations of $4 \mathrm{mmol} / \mathrm{liter}$. In a linked twin-pot assay, inoculation with 2,4-di-tert-butylphenol or 3,3-dimethyloctane reduced the number of nematodes in the soil (by 54.9 and $70.6 \%$, respectively), the number of galls (by 53.7 and $52.4 \%$ ), and the number of $M$. incognita in root tissues (by 67.5 and $36.3 \%$ ). BCM2 colonization in tomato roots affected the composition of root exudates, increasing the secretion of substances that appear to be repellent, thus decreasing $M$. incognita J2 infection of roots.
\end{abstract}

Root-knot disease is caused by plant-parasitic root-knot nematodes (RKN; Meloidogyne spp.), which can infect a broad range of plant species worldwide and cause crop losses estimated at more than $\$ 157$ billion annually (Abad and Gouzy 2008). At present, pesticides, such as fosthiazate and 1,3-dichloropropene, are widely used to control RKN, as they are simple to use and work quickly (Schneider and Rosskopf 2003; Xiong and Zhou 2015). However, pesticide use may leave toxic residues in the environment and promote the evolution of resistance. As concerns for the environment and public safety mount, many highly toxic nematicides have been withdrawn or their use restricted in developed countries (Schneider and Rosskopf 2003). Therefore, there is an urgent need for environmentally acceptable and sustainable control strategies.

Endophytic bacteria colonize plant internal tissues at various stages of the life cycle and do not cause disease. Plant bacterial endophytes may have beneficial effects on their host by promoting plant growth and controlling the development of plant diseases (Backman and Sikora 2008). Researchers are now uncovering the potential of endophytic bacteria to control RKN, suggesting the feasibility of future biocontrol (Lopes et al. 2018; Sikora et al. 2007; Su et al. 2017). Bacillus has been studied extensively as endophytic bacteria for control of RKN, with Bacillus pumilus, B. brevis, B. megaterium, $B$. mycoides, $B$. licheniformis, and $B$. thuringiensis reported to successfully

${ }^{\dagger}$ Corresponding author: S.-Z. Yan; yanshuzhen@njnu.edu.cn

X. Li and H.-J. Hu contributed equally to this work and should be considered co-first authors.

Funding: This work was supported by the National Sanitation Foundation of Jiangsu Province, China, no. BK2012848.

The authors declare no conflict of interest.

Accepted for publication 14 January 2019.

() 2019 The American Phytopathological Society reduce the number of galls and egg masses caused by $M$. incognita (Hallmann et al. 2009; Yu and Xiong 2015). It is thought that endophytic bacteria control RKN by altering host-nematode interactions, thereby inhibiting RKN during the early stages of root invasion (Sikora et al. 2007). Bacterial endophytes may directly affect RKN by producing toxins that kill or inhibit RKN activity (Niu and Tian 2011; Zhang and Peng 2012), or by displacing RKN by colonizing their feeding sites (Hallmann et al. 1998). Endophytes may also indirectly affect RKN by altering the composition of root exudates (Singh and Sahu 2013) or inducing plant resistance (Adam et al. 2014; Hashem and Abo-Elyousr 2011; Sikora et al. 2007; Singh and Sahu 2013; Vos and Schouteden 2013).

Root exudates are comprised of organic and inorganic substances that plant roots release to the rhizosphere through various means. Both soil conditions and numerous biological factors can influence root exudate composition. Some RKNs require the presence of specific factors in host plant exudates to stimulate hatching and development; therefore, a change in root exudates may affect hatching and the ability to identify suitable hosts (Sikora et al. 2007). As previously reported, the presence of tannins, flavonoids, glycosides, and fatty acids in root exudates can modulate the behavior of RKN second-stage juveniles (J2) before infestation occurs (Bais and Weir 2006; Chitwood 2002). It has also been reported that chemicals such as the small lipophilic molecules present in the root exudates of tomato and rice plants can inhibit RKN stylet thrusting and locomotor ability, and produce inhibitory effects on the RKN (Dutta and Powers 2012).

In a previous study, the endophyte Bacillus cereus BCM2 was found to have a strong ability to colonize tomato roots, and also to inhibit infection by $M$. incognita $\mathrm{J} 2$; inoculation with BCM2 was associated with a $81.2 \%$ reduction in the number of galls and a $75.6 \%$ reduction in nematode egg mass (Hu and Chen 2017). In order to further explore how BCM2 colonization may change the composition of tomato root exudates and control $M$. incognita infestations, a paired, linked twin-pot assay was used to verify that BCM2 inoculation in tomato plants may have a repellent effect on $M$. incognita $\mathrm{J} 2$. Root exudates from tomato plants that were inoculated with BCM2 were 
compared with those from plants treated with sterile water only; root exudate components that differed in abundance between treatments were further analyzed and their effects on $M$. incognita $\mathrm{J} 2$ tested.

\section{Materials and Methods}

Preparation of Bacillus cereus BCM2. The Bacillus cereus strain BCM2 was isolated from strawberry fruits grown in Nanjing, in Jiangsu Province, China (Hu and Chen 2017), and stored at $-80^{\circ} \mathrm{C}$ at the Institute of Microbial Resources, part of the College of Life Sciences at Nanjing Normal University. BCM2 was grown on nutrient agar plates containing beef extract and peptone at $28^{\circ} \mathrm{C}$ for $48 \mathrm{~h}$. Individual isolates were inoculated into $100 \mathrm{ml}$ of beef extractpeptone broth and incubated on a rotary shaker at $28{ }^{\circ} \mathrm{C}$ and $160 \mathrm{rpm}$ for $48 \mathrm{~h}$. Cultures were centrifuged at 5,000 rpm for 5 min to collect the bacteria. Pellets were then washed three times with sterile water before resuspending in sterile water. The bacterial suspension density was quantified at $10^{8} \mathrm{CFU} / \mathrm{ml}$ (colony forming units): the $\mathrm{OD}_{600}$ (optical density at $600 \mathrm{~nm}$ ) of the suspension was 0.480 , and the cell density was $2.25 \times 10^{8} \mathrm{CFU} / \mathrm{ml}$.

Preparation of second-stage Meloidogyne incognita juveniles (J2). Dahong tomato Hezuo903 seeds (Solanum lycopersicum) were obtained from the Tomato Institute of Shanghai. Tomato plants were grown at temperatures of $28^{\circ} \mathrm{C}$ (day: $14 \mathrm{~h}$ ) and $20^{\circ} \mathrm{C}$ (night: $10 \mathrm{~h}$ ), and a constant $75 \%$ humidity. Each plant grew in a pot $(15 \mathrm{~cm}$ in diameter and $14 \mathrm{~cm}$ in height) that contained $1,000 \mathrm{~g}$ of a 1:1 substrate mixture of silica sand and potting soil. The soil organic matter content was determined as $80.25 \mathrm{~g} \mathrm{~kg}^{-1}$ by measuring the mass lost after treatment at $550^{\circ} \mathrm{C}$ for $5 \mathrm{~h}$ (Bao et al. 2011), and the total nitrogen, available phosphorus, and available potassium were determined as $3.58,10.25$, and $138.23 \mathrm{~g} \mathrm{~kg}^{-1}$ (Jie et al. 2006). M. incognita was provided by Professor Hongmei Li from the Department of Plant Pathology at Nanjing Agricultural University. The $M$. incognita population was maintained on tomato plants. Eight weeks after inoculation, $M$. incognita egg masses were hand-picked from diseased tomato roots. Egg masses were then dipped in $0.5 \% \mathrm{NaOCl}$ for $2-3 \mathrm{~min}$ and rinsed three times with sterile water (Hussey and Barker 1973). The eggs were incubated for 2-5 days at $25^{\circ} \mathrm{C}$, and then $M$. incognita $\mathrm{J} 2$ were collected using a modified Baerman funnel method (Gray 1984; Hu and Chen 2017).

Effects of $\mathrm{BCM} 2$ on $M$. incognita $\mathrm{J} 2$ in a linked twin-pot assay. To test for effects of BCM2 on M. incognita J2, Adam's (Adam et al. 2014) linked twin-pot testing assay was modified according to experimental requirements. Each pot measured $7 \times 7 \times 8 \mathrm{~cm}$, and pairs of pots were connected with a tee-shaped piece of piping. The inner diameter of the connecting tee was $2 \mathrm{~cm}$, the connecting arm was $5 \mathrm{~cm}$ long, and the inoculation hole was $4 \mathrm{~cm}$ deep. The two pots (including the connecting tee) were filled to the brim with a soil mixture (a 1: 1 mix of silica sand: potting soil). In order to rule out the effects of other bacteria in the soil, the soil used in all the experiments was sterilized. A single 2-week-old tomato seedling was planted in each pot. Seven days after transplanting, one-half of the tomato seedlings in pots (i.e., one per pair) were inoculated with $10 \mathrm{ml}$ of a BCM2

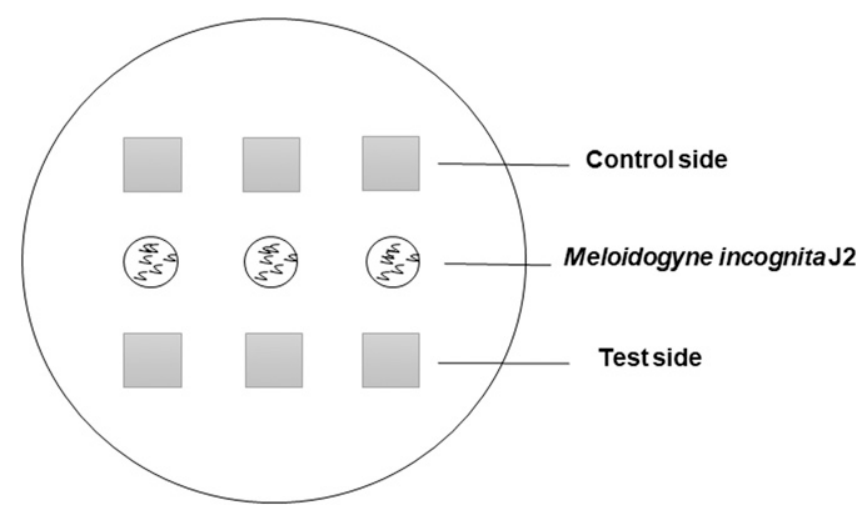

Fig. 1. Schematic of the water agar plates for the Meloidogyne incognita second-stage juveniles (J2) chemotaxis assay. suspension with $\mathrm{OD}_{600}=0.5$ (i.e., the concentration of bacterial cells was $2.25 \times 10^{8} \mathrm{CFU} / \mathrm{ml}$ ), while the other half received $10 \mathrm{ml}$ of sterile water. After 3 days, 1,000 J2 in $1 \mathrm{ml}$ of sterile water were added to the inoculation hole of each pair. Each treatment was replicated 10 times and nematode infestation was quantified 40 days after inoculation. First, the number of galls was counted. Then, using Fan's method (Fan and $\mathrm{Hu} 2014$ ), the number of nematodes per gram of root tissue was estimated, and the number of nematodes per $100 \mathrm{~g}$ of the rhizosphere determined following Affokpon's method (Affokpon and Coyne 2011). The height, fresh weight, and dry weight of the tomato plants were also measured.

Collection and concentration of tomato root exudates. Twoweek-old tomato seedlings were planted in pots, each containing $1,000 \mathrm{~g}$ of soil (as described above). Plants were treated with either (i) $10 \mathrm{ml}$ of sterile water as a control, or (ii) $10 \mathrm{ml}$ of BCM2 suspension. Fifteen days postinoculation (dpi), the tomato seedlings were removed from the pots, being careful not to damage the roots. The roots were washed and put into new plastic cups; each plastic cup received five seedlings and $100 \mathrm{ml}$ of sterile distilled water to submerge the roots. Cups were maintained at $28^{\circ} \mathrm{C}$ (day: $14 \mathrm{~h}$ ) and $20^{\circ} \mathrm{C}$ (night: $10 \mathrm{~h}$ ), and $75 \%$ relative humidity for $48 \mathrm{~h}$ (Wang et al. 2005). Each treatment was replicated six times. Root exudates were then filtered sequentially through $0.45 \mu \mathrm{m}$ and $0.22 \mu \mathrm{m}$ filters to remove impurities, such as soil and bacteria. The filtrate was collected and an equal volume of cyclohexane was added for a 24-h extraction. After the extraction was complete, the aqueous and organic phases were volatilized separately.

Effects of tomato root exudates on $M$. incognita $\mathrm{J} 2$ in vitro and a linked twin-pot assay. A total of $200 \mu \mathrm{l}$ of sterile water and $200 \mu \mathrm{l}$ of DMSO (solubilization solution) were used to redissolve the root exudates volatilized in the previous step. As shown in Figure 1, $1 \mathrm{~cm}^{2}$ pieces of filter paper were placed on a $0.5 \%$ water agar plate; pieces were spaced uniformly $2 \mathrm{~cm}$ apart. To test for the chemotaxis effects of tomato root exudates on $\mathbf{J} 2,20 \mu \mathrm{l}$ of the redissolved root exudates were added to each piece of filter paper. Thus, treatments included (i) tomato root exudates from plants previously inoculated with $\mathrm{BCM} 2$, and (ii) tomato root exudates from plants previously inoculated with sterile water (CK). Between each filter paper pair, 100 $\mathrm{J} 2$ suspended in $20 \mu \mathrm{l}$ of sterile water were added to the plate, and plates were then incubated at $28^{\circ} \mathrm{C}$. The movement of $\mathrm{J} 2$ on the water agar plate was observed after $48 \mathrm{~h}$ (Hu and Chen 2017). There were 10 replicates of each treatment.

After the observation period, each filter paper piece was transferred to a 2-ml centrifuge tube; $1 \mathrm{ml}$ of sterile water was added to each tube before shaking at 1,500 rpm for $10 \mathrm{~min}$. After allowing $10 \mathrm{~min}$ of natural sedimentation, the filter paper was discarded and the tube centrifuged at $12,000 \mathrm{rpm}$ for $1 \mathrm{~min}$. The number of $\mathrm{J} 2$ in each sample was counted under a light microscope. Next, the J2 under the filter paper were counted using an inverted microscope. The total number of nematodes that migrated to the source was counted on the filter paper as well as from under the filter paper.

Tomato seedlings were grown in a linked twin-pot assay as previously described. To one plant per pair, $10 \mathrm{ml}$ of root exudates from plants inoculated with BCM2 were added, and to the other plant, $10 \mathrm{ml}$ of root exudates from plants inoculated with sterile water (CK) were added. Root exudates were added every 3 days, for a total of three applications. After the first application, 1,000 J2 were added to the connecting tee between the pots; nematode infestation was quantified 40 days after inoculation. Each treatment was replicated three times.

Component detection and content determination of root exudates by GC. An Agilent 7890A Gas Chromatograph equipped with an HP-5 capillary column was used to analyze the tomato root exudates. The carrier gas was helium with a flow rate of $1 \mathrm{ml} / \mathrm{min}$ in split/ splitless mode. For each sample, $1 \mu l$ of the tomato root exudates was introduced directly into the front inlet of the gas chromatograph and desorbed at $220^{\circ} \mathrm{C}$. The oven temperature varied as follows: from $60^{\circ} \mathrm{C}$ to $140^{\circ} \mathrm{C}$ at a rate of $10^{\circ} \mathrm{C} / \mathrm{min}$ for $2 \mathrm{~min}$, from $140^{\circ} \mathrm{C}$ to $220^{\circ} \mathrm{C}$ at $15^{\circ} \mathrm{C} / \mathrm{min}$ for $2 \mathrm{~min}$, and then from $220^{\circ} \mathrm{C}$ to $240^{\circ} \mathrm{C}$ at $20^{\circ} \mathrm{C} / \mathrm{min}$ for $10 \mathrm{~min}$. 
To examine the effects of BCM2 versus sterile water inoculation on the composition of tomato root exudates, the chromatograms of samples from these two treatments were evaluated. Peak area was compared with determined differentially abundant components that were then selected for further analysis.

Identification of active constituents by GC-MS. An Agilent 6890/5975 Gas Chromatograph/Mass Spectrometer equipped with a DB-5 capillary column was used to identify the active components of the tomato root exudates. The carrier gas was helium with a flow rate of $1 \mathrm{ml} / \mathrm{min}$ in split/splitless mode. For each sample, $2 \mu \mathrm{l}$ of the tomato root exudates was introduced directly into the front inlet of the gas chromatograph and desorbed at $280^{\circ} \mathrm{C}$. Oven temperature increased from $60^{\circ} \mathrm{C}$ to $300^{\circ} \mathrm{C}$ at a rate of $15^{\circ} \mathrm{C} / \mathrm{min}$. The temperature of the ion trap was $230^{\circ} \mathrm{C}$. Identification of key chemical components within the tomato root exudates was based on comparisons between the mass spectrum of a given substance and standards provided by the U.S. National Institute of Standards and Technology (NIST) GC-MS system database.

Standard calibration by GC. Putative active components identified in the previous step were purchased at the Dr. Ehrenstorfer GmbH chemical store, using chemical names as provided by the NIST database. These were then analyzed by GC, under the exact same conditions described previously, to confirm component identity.

Effects of standards on movement of $M$. incognita $\mathrm{J} 2$ in vitro and in a linked twin-pot assay. On the basis of observed quantities of components of interest in the root exudates, standards were prepared for each at three concentrations: $0.25,1.0$, and $4.0 \mathrm{mmol} /$ liter. In the preparation, the agent was dissolved with absolute ethanol, and distilled water was slowly added until the desired concentration was reached. The final concentration of ethanol was $1.0 \%$; hence, $1.0 \%$ ethanol was used as the control (CK).

To test the repellence effects of the standards on $\mathrm{J} 2$, all three concentrations of each standard were tested on water agar plates, as shown in Figure 2; $20 \mu \mathrm{l}$ of the prepared standards were added to area I. $100 \mathrm{~J} 2$ suspended in $20 \mu \mathrm{l}$ of sterile water were added to the center of the plate, and plates were then incubated at $28^{\circ} \mathrm{C}$. The movement of $\mathrm{J} 2$ on the water agar plate was observed after $48 \mathrm{~h}(\mathrm{Hu}$ and Chen 2017). The number of J2 in areas I, II, III, and IV were counted under an inverted microscope. Each treatment was replicated six times.

Tomato seedlings were grown in a linked twin-pot assay as previously described. To one plant per pair, $10 \mathrm{ml}$ of the $4 \mathrm{mmol} / \mathrm{liter}$ standard was added, while to the other plant, $1.0 \%$ ethanol served as a control. Standards were added every 3 days, for a total of three applications. After the first application, 1,000 J2 were added to the inoculation hole between pots; nematode infestation was quantified 40 days after inoculation. Each treatment was replicated six times.

Statistical analysis. Data were analyzed using SPSS version 17.0, and the mean \pm standard deviation (SD) provided for quantities of interest. One-way analyses of variance and Duncan's multiple-range tests were employed to test for differences among means. An alpha level of 0.05 was used to determine statistical significance.

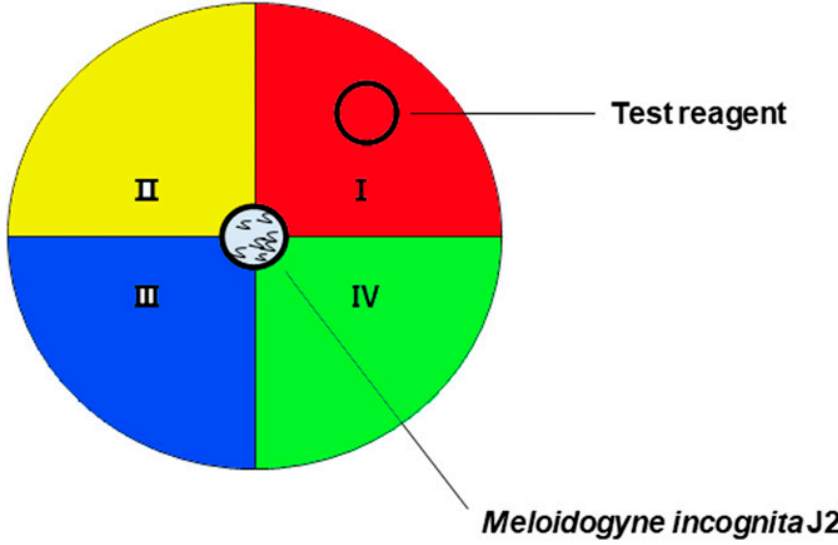

Fig. 2. Schematic of the water agar plates for the Meloidogyne incognita repellency assay with second-stage juveniles (J2).

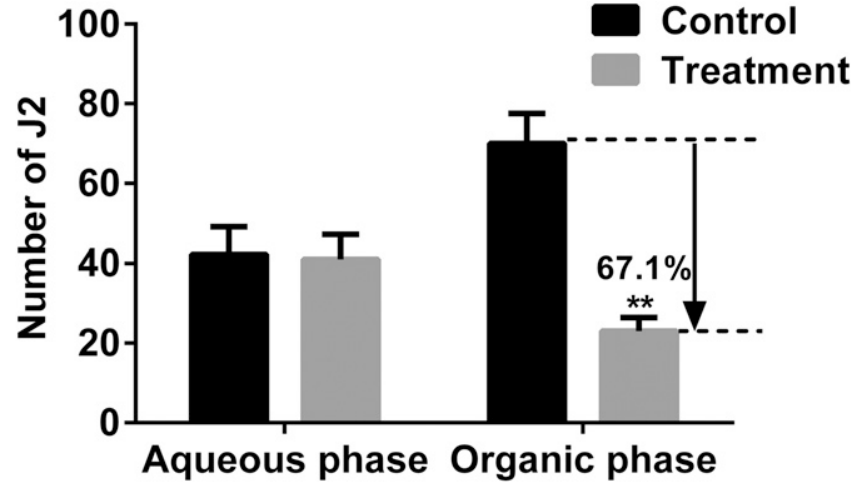

Fig. 4. Effects of tomato root exudates on Meloidogyne incognita second-stage juveniles (J2) in vitro. Exudates were prepared by cyclohexane leaching. Double asterisks $\left(^{* *}\right)$ indicate a significant reduction $(P \leq 0.01, n=10)$ from treatment to control.

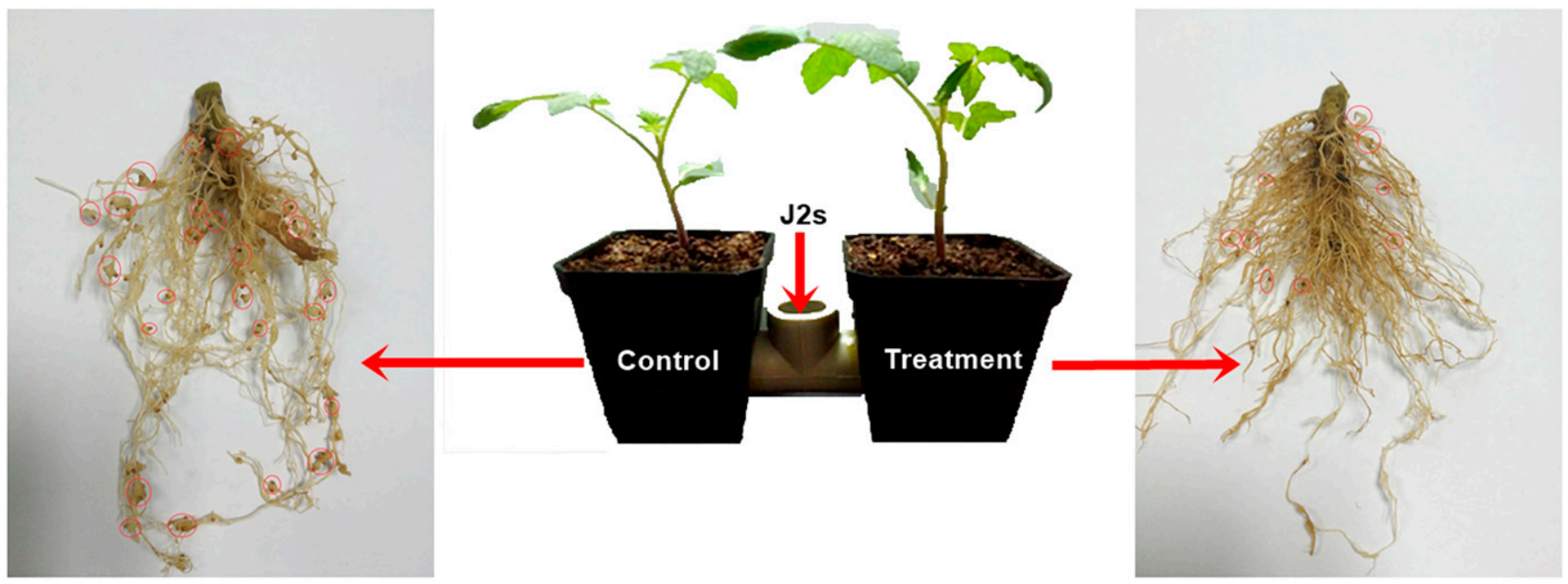

Fig. 3. Schematic of the linked twin-pot assay used to determine the influence of Bacillus cereus BCM2 colonization on Meloidogyne incognita. 


\section{Results}

Effects of BCM2 on M. incognita $\mathrm{J} 2$ in a linked twin-pot assay. As shown in Table 1, within plant pairs, the number of $\mathrm{J} 2$ in the rhizosphere was $62.3 \%$ lower on the side inoculated with BCM2 versus the sterile water side; thus, more $\mathrm{J} 2$ moved to the side of the tomato plant inoculated with sterile water, indicating that $M$. incognita J2 were either less attracted to or were repelled from BCM2 colonized roots. A schematic of the linked twin-pot assay and photos of representative root infections are provided in Figure 3. Infestation by $M$. incognita J2 was significantly reduced by the presence of BCM2, when measured as either the number of galls or the abundance of $M$. incognita juveniles in root tissue (a $45.0 \%$ and $58.7 \%$ decrease, respectively), indicating that BCM2 colonization inhibited $\mathrm{J} 2$ infection.

Effects of tomato root exudates on $M$. incognita $\mathrm{J} 2$ in vitro and a linked twin-pot assay. The results of the water agar plate experiment are shown in Figure 4. The organic phase of root exudates from tomato plants inoculated with BCM2 had $67.1 \%$ fewer J2 compared

Table 1. Effect of colonization by endophytic bacteria, Bacillus cereus BCM2, on Meloidogyne incognita second-stage juveniles (J2) and growth of tomato plants infected with $M$. incognita in a paired linked twin-pot assay ${ }^{\mathrm{a}}$

\begin{tabular}{lccc}
\hline & BCM2 & CK & $\begin{array}{c}\text { Rate of } \\
\text { change (\%) }\end{array}$ \\
\hline Juveniles/100 g soil & $6.31 \pm 7.93^{* *}$ & $16.75 \pm 13.83$ & -62.3 \\
Galls/g root & $7.19 \pm 3.05^{*}$ & $13.07 \pm 7.58$ & -45.0 \\
Juveniles/g root & $11.04 \pm 5.71^{* *}$ & $26.70 \pm 18.77$ & -58.7 \\
Length (cm) & $18.00 \pm 3.32$ & $16.09 \pm 2.97$ & 11.9 \\
Fresh weight (g) & $4.00 \pm 0.98$ & $3.29 \pm 0.70$ & 21.6 \\
Dry weight $(\mathrm{g})$ & $0.67 \pm 0.13$ & $0.62 \pm 0.09$ & 8.1 \\
\hline
\end{tabular}

${ }^{\text {a }}$ Data are presented as the mean \pm standard deviation $(\mathrm{n}=10)$. Values followed by an asterisk $\left(^{*}\right)$ are significantly different at $P \leq 0.05$ according to one-way ANOVAs and Duncan's multiple-range test. Double asterisks $(* *)$ indicate significant differences $(P \leq 0.01)$ between treatment and control (no endophytic bacteria).

Table 2. Effects of tomato root exudates on Meloidogyne incognita secondstage juveniles (J2) in a linked twin-pot assay

\begin{tabular}{lccc}
\hline & Treatment $^{\mathbf{a}}$ & CK $^{\mathbf{b}}$ & $\begin{array}{c}\text { Inhibition } \\
\text { rate (\%) }^{\mathbf{c}}\end{array}$ \\
\hline Juveniles/100 g soil & $21.67 \pm 12.47^{*}$ & $37.33 \pm 15.63$ & 42.0 \\
Galls/g root & $78.17 \pm 47.23^{*}$ & $138.04 \pm 49.28$ & 43.3 \\
Juveniles/g root & $65.73 \pm 34.26^{*}$ & $125.55 \pm 8.75$ & 47.7 \\
\hline
\end{tabular}

a Treatment refers to the application of root exudates from plants inoculated with Bacillus cereus BCM2.

${ }^{\mathrm{b}} \mathrm{CK}$ refers to exudates from plants inoculated with sterile water.

${ }^{\mathrm{c}}$ Data are presented as the mean \pm standard deviation $(\mathrm{n}=3)$. Values followed

by an asterisk $(*)$ are significantly different at $P \leq 0.05$ according to oneway ANOVAs and Duncan's multiple-range tests. with the control. The inorganic phase of the root exudates had no such inhibitory effect on $M$. incognita $\mathrm{J} 2$.

In the linked twin-pot assay, the number of M. incognita $\mathrm{J} 2$ was $42.0 \%$ lower in soils with root exudates from BCM2 plants compared with the control. Similarly, the number of galls and the number of $M$. incognita juveniles counted in root tissues were reduced by $43.3 \%$ and $47.7 \%$, respectively, in the treatment versus control (Table 2).

Analysis of root exudates and standard calibration. Gas chromatograms of tomato root exudates inoculated with BCM2 and sterile water were compared and analyzed. The results are shown in Table 3. Compared with sterile water, inoculation with BCM2 led to differences in the composition and content of tomato root exudates; seven substances were significantly more abundant in the $\mathrm{BCM} 2$ treatment exudates. Of these, substance 2 is a secretion induced specifically by BCM2; considering the other six compounds, substance 7 showed the greatest increase in the treatment versus control, followed by substance $1,5,6,3$, and 4 .

The above anonymous compounds were identified using GC-MS; substance 1 was identified as 3,3-dimethyloctane, substance 4 as n-tridecane, substance 6 as 2,4-di-tert-butylphenol, and substance 7 as n-heneicosane. There was no match for substances 2,3 , and 5 in the library. Gas chromatographic examination of the chemical standards (Dr. Ehrenstorfer $\mathrm{GmbH}$ ) showed that the retention times of 3,3-dimethyloctane, 2.4-di-tert-butylphenol, and n-tridecane were the same as those of substances 1,4 , and 6 . However, the retention time of substance 7 was not consistent with any tested n-heneicosane, indicating that substance 7 is not n-heneicosane. Chromatograms of the three standards are shown in Figure 5 (B-D).

Verification of repellent effects on $M$. incognita $\mathrm{J} 2$ with the three standards. The results of the water agar plate experiments are presented in Figure 6 for all three standards. At a concentration of $4 \mathrm{mmol} /$ liter, 3,3-dimethyloctane had a significant repelling effect on M. incognita $\mathrm{J} 2$, more $\mathrm{J} 2$ moved to area III, but as its concentration decreased, so did its repellent effect. At the lowest concentration of $0.25 \mathrm{mmol} /$ liter, the addition of 3,3-dimethyloctane even had an attractive effect on $M$. incognita J2 (Fig. 6A). n-Tridecane repelled $M$. incognita $\mathrm{J} 2$ at a concentration of $4 \mathrm{mmol} / \mathrm{liter}$; at lower concentrations, it strongly attracted $M$. incognita J2 (Fig. 6B). All concentrations of 2,4-di-tert-butylphenol repelled $M$. incognita $\mathrm{J} 2$; however, at $0.25 \mathrm{mmol} / \mathrm{liter}$, its repellent effect was weaker (Fig. $6 \mathrm{C}$ ). Thus, repellent effects of the above substances on $M$. incognita J2 showed a dose-response relationship.

The results of the linked twin-pot assay are presented in Figure 7 for all three standards. The application of 3,3-dimethyloctane, n-tridecane, and 2,4-di-tert-butylphenol resulted in a 52.4, 31.8, and 53.7\% reduction, respectively, in the number of root galls developing as compared with controls (Fig. 7A). Similarly, the application of 3,3-dimethyloctane and 2,4-di-tert-butylphenol resulted in a 36.3 and $67.5 \%$ reduction in the number of M. incognita juveniles counted in root tissue as compared with controls (Fig. 7B). Finally, 3,3-dimethyloctane, n-tridecane, and 2,4-di-tert-butylphenol treatments reduced the number of M. incognita juveniles counted in the soil by $70.6,61.1$, and $54.9 \%$, respectively, compared with controls (Fig. 7C).

Table 3. Differentially abundant components of tomato root exudates after inoculation with Bacillus cereus BCM2

\begin{tabular}{|c|c|c|c|c|c|c|}
\hline & $\begin{array}{l}\text { Retention } \\
\text { time }\end{array}$ & $\begin{array}{l}\text { Peak area of } \\
\text { treatment }^{\mathrm{a}}\end{array}$ & $\begin{array}{c}\text { Peak area } \\
\text { of } \mathbf{C K}^{\mathbf{b}}\end{array}$ & $\begin{array}{c}\text { Rate of } \\
\text { increase }(\%)\end{array}$ & $\begin{array}{l}\text { Concentration in } \\
\text { treatment }(\mathrm{mmol} / \mathrm{L})\end{array}$ & $\begin{array}{c}\text { Concentration } \\
\text { in } \mathrm{CK}(\mathrm{mmol} / \mathrm{L})\end{array}$ \\
\hline Substance $1^{\mathrm{c}}$ & 16.222 & 131.7892 & 74.3513 & 77.3 & 0.5566 & 0.3536 \\
\hline Substance 2 & 19.275 & 16.5023 & 0.0000 & 100.0 & & \\
\hline Substance 3 & 21.708 & 112.0962 & 72.3844 & 54.9 & & \\
\hline Substance $4^{\mathrm{d}}$ & 22.585 & 58.1952 & 37.8313 & 53.8 & 0.2263 & 0.1375 \\
\hline Substance 5 & 25.313 & 135.2870 & 76.8428 & 76.1 & & \\
\hline Substance $6^{\mathrm{e}}$ & 25.583 & 238.3306 & 148.1143 & 60.9 & 0.0040 & 0.0028 \\
\hline Substance 7 & 25.937 & 58.5922 & 32.9139 & 78.0 & & \\
\hline
\end{tabular}

a Treatment refers to the application of root exudates from plants inoculated with BCM2.

${ }^{\mathrm{b}} \mathrm{CK}$ refers to exudates from plants inoculated with sterile water.

c Substance 1 represents 3,3-dimethyloctane.

d Substance 4 represents n-tridecane.

e Substance 6 represents 2,4-di-tert-butylphenol. 


\section{Discussion}

In recent years, researchers have isolated a large number of endophytic bacteria that promote plant growth and reduce RKN infestations (Hu and Chen 2017; Pradana et al. 2015; Su and Shen 2017; Vetrivelkalai et al. 2010). However, little is known regarding the mechanisms by which endophytic bacteria control RKN. There were some studies suggesting that endophytic bacteria may alter host plant root exudates to avoid infection by $M$. incognita, but few studies have identified the chemical changes that occur (Singh and Sahu 2013). A significant stimulation of root exudates by endophytes was detected by previous studies (Van Hecke et al. 2005; Omacini et al. 2012). The authors suggested that the significant change in the root exudates may affect the local biotic interactions, such as enemy release, disruption, or promotion of other biotic associations. It was observed that the root exudates from Fusarium moniliforme Fe14-treated plants were either less attractive or had repellent effect on nematode movement (Le and Padgham 2016). In this work, the effects of BCM2 colonization on the composition of tomato root exudates was evaluated and potential mechanisms for BCM2-mediated $M$. incognita control explored.
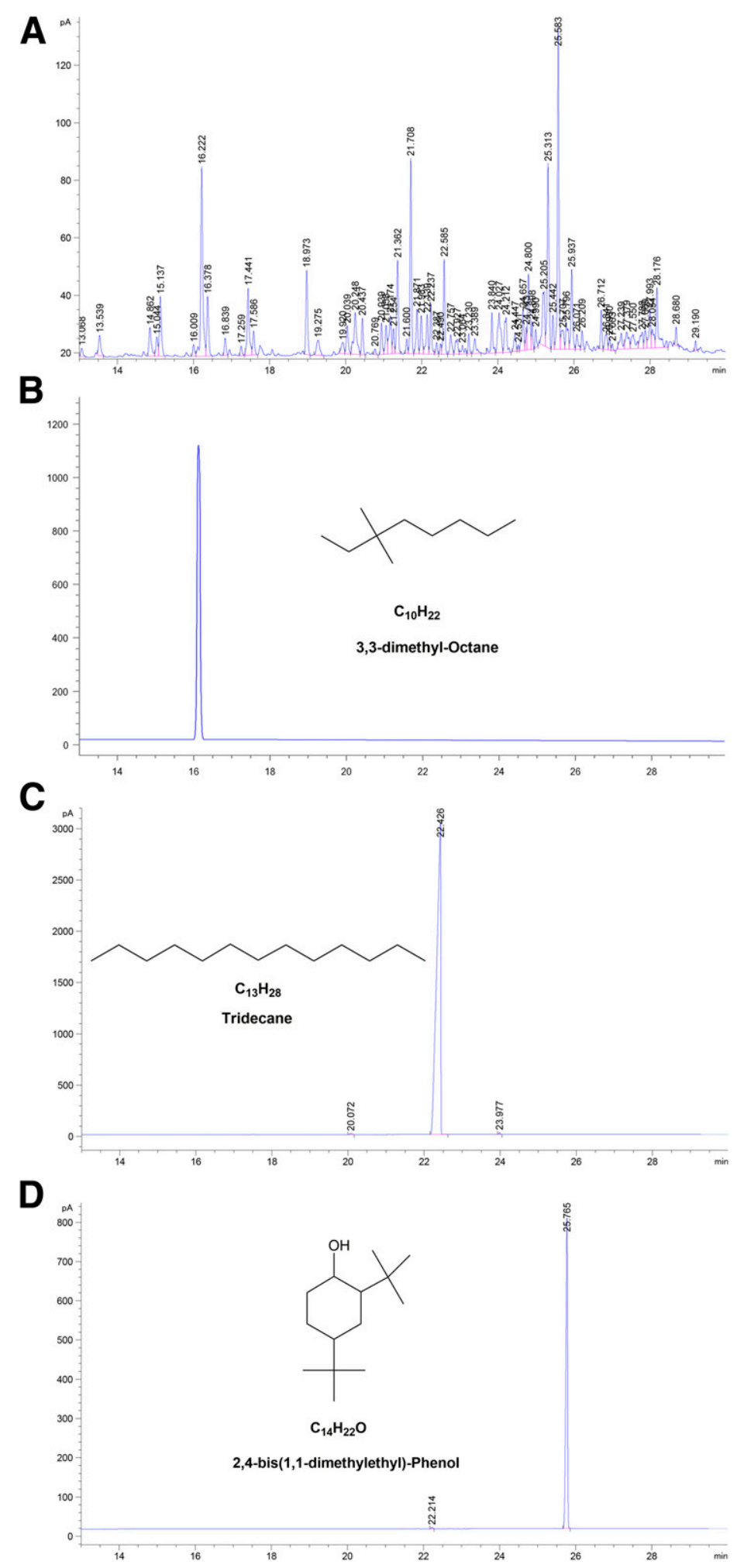

Fig. 5. GC chromatogram of (A) the root exudates from plants inoculated with Bacillus cereus BCM2, (B) 3,3-dimethyloctane, (C) n-tridecane, and (D) 2,4-di-tert-butylphenol. 
In a previous study, colonization of tomato roots by the endophyte B. cereus BCM2 was found to reduce the number of galls by $81.2 \%$ in vitro, suggesting a strong inhibitory effect on $M$. incognita $\mathrm{J} 2(\mathrm{Hu}$ and Chen 2017). In the linked twin-pot assay of this study, $M$. incognita $\mathrm{J} 2$ tended to move toward the control tomato plant, not inoculated with $\mathrm{BCM} 2$, indicating that BCM2 colonization may lower $M$. incognita invasion. Parasitic nematodes typically search for hosts through specific odors (Baiocchi et al. 2017). For example, one study found that $\alpha$-pinene, $\beta$-pinene, and longifolene strongly attracted pine wood nematodes (Zhao et al. 2007). In this experiment, tomato plants inoculated with endophytic BCM2 were less attractive, or even repellent, to RKN J2. Similarly, Bacillus megaterium colonization of
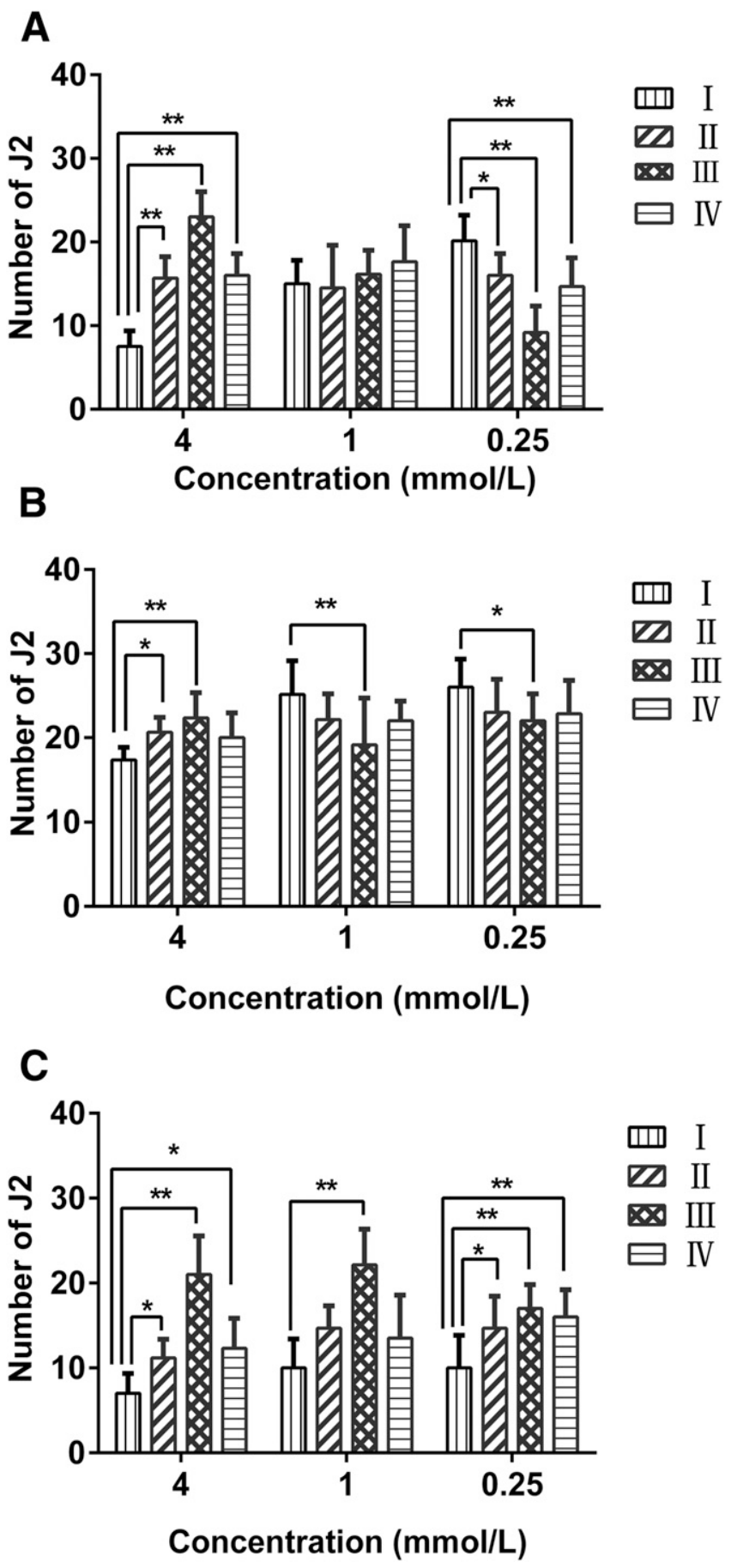

Fig. 6. In vitro movement of Meloidogyne incognita second-stage juveniles (J2) to different concentrations of (A) 3,3-dimethyloctane, (B) n-tridecane, and (C) 2,4-di-tertbutylphenol. Asterisks denote significant differences between areas II, III, IV and area $\mathrm{I}$; $\left(^{*}\right)$ indicates $P \leq 0.05(n=6)$, while $\left(^{* *}\right)$ indicates $P \leq 0.01(n=6)$. rice roots reduced migration of Meloidogyne graminicola to the root zone by $60 \%$ (Padgham and Sikora 2007).

In both water agar plate and linked twin-pot assay, the root exudates of tomatoes colonized by BCM2 attracted fewer $\mathrm{J} 2$ than tomato roots not inoculated with BCM2 (i.e., controls). Therefore, BCM2 colonization in tomato roots seems likely to have changed the metabolite composition of root exudates, making them less attractive or
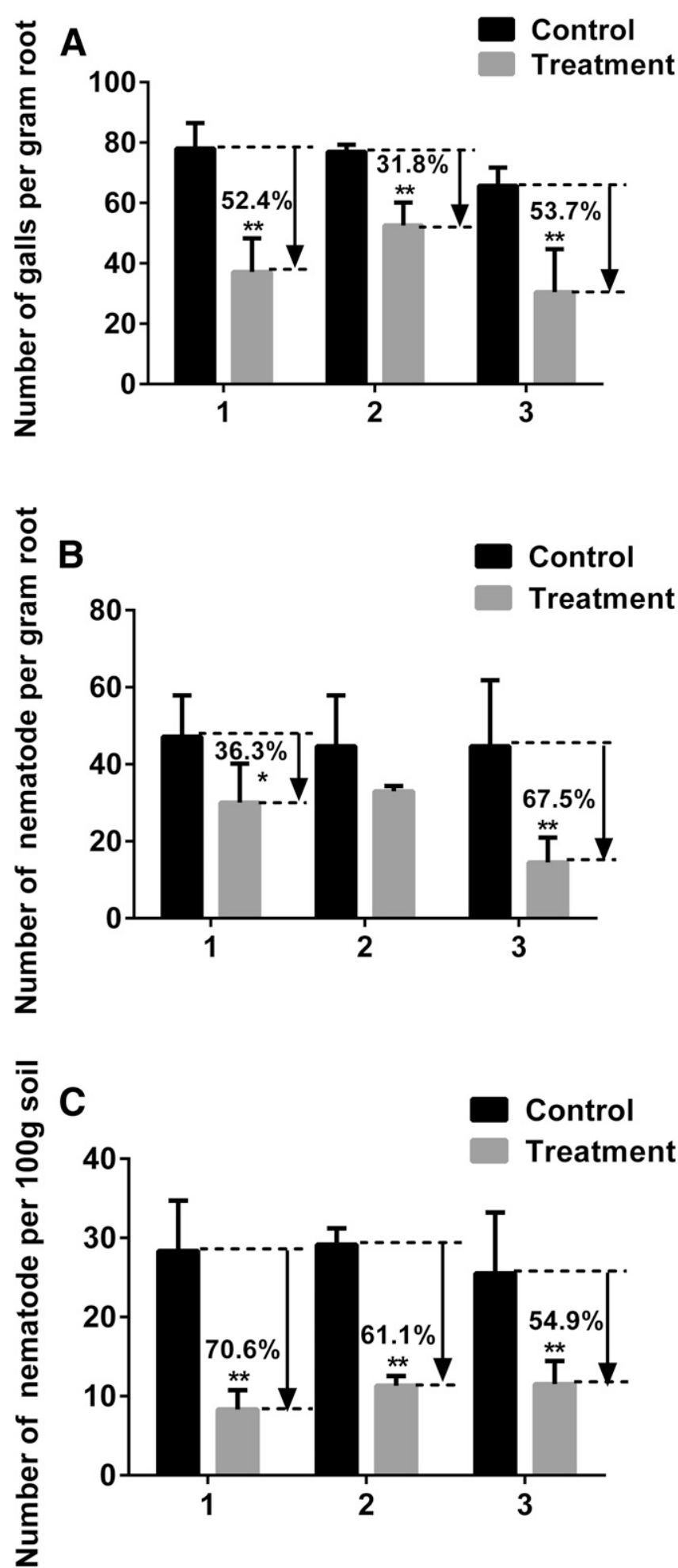

Fig. 7. Effects of three chemical standards on Meloidogyne incognita second-stage juveniles (J2), where 1, 2, and 3 represent 3,3-dimethyloctane, n-tridecane, and 2,4-di-tert-butylphenol, respectively: (A) Number of galls per gram of root tissue, (B) number of $M$. incognita juveniles per gram of root tissue, and (C) number of $M$. incognita juveniles per $100 \mathrm{~g}$ of soil. Asterisks denote significant differences between treatment and control; $\left({ }^{*}\right)$ indicates $P \leq 0.05(n=6)$, while $\left(^{* *}\right)$ indicates $P \leq 0.01(n=6)$. 
repellent to J2. Similarly, it has been reported that most $M$. incognita J2 chose to infect control plants not treated with Fusarium oxysporum (Sikora and Dababat 2007). The authors hypothesized that the root exudates of plants colonized by $F$. oxysporum were less attractive to, or had a repulsive effect on, $M$. incognita, but they did not identify the substance responsible.

While components of root exudates are used by plant-parasitic nematodes to orient to their hosts (Niu and Huang 2010), some components of exudates have been shown to deter nematodes. Small lipophilic molecules in the root exudates of tomato and rice plants were found to inhibit RKN stylet thrusting and locomotor ability, producing repellent effects on the RKN (Dutta and Powers 2012). Analysis of three tomato root exudate samples with varying levels of nematode resistance found the abundance of esters and phenolic compounds in the exudates was greater in highly and moderately resistant plants compared with highly susceptible ones. Among the tested compounds, dibutyl phthalate repelled $M$. incognita J2 (Yang and Zhou 2016). In this study, the composition of tomato root exudates inoculated with BCM2 differed from those not inoculated, with the concentration of 3,3-dimethyloctane, n-tridecane, and 2,4-di-tertbutylphenol significantly higher in the former, as identified by GC-MS and standard calibration. Furthermore, 2,4-di-tert-butylphenol, 3,3dimethyloctane, and to a lesser extent $n$-tridecane appear to produce avoidance behaviors on $M$. incognita $\mathrm{J} 2$ on water agar plates in a dosedependent manner. Similarly, in the linked twin-pot assay, the application of 2,4-di-tert-butylphenol and 3,3-dimethyloctane to tomato roots reduced the number of $M$. incognita found in the soil as well as the infection rate, as measured by the number of galls and juveniles present in root tissue. Therefore, 2,4-di-tert-butylphenol and 3,3-dimethyloctane may be the components that appear to repel M. incognita after inoculation with BCM2.

2,4-di-tert-Butylphenol has been reported to have antioxidant activity and fumigation bactericidal activity (Yoon and Tae-Sook 2006; Tayung et al. 2010); it can inhibit mycelial growth and spore formation of Phytophthora capsici and stimulate fruit ripening (Sang et al. 2011; Sang and Kim 2012). At the same time, ntridecane has been reported to have toxicities and repellencies to three pests, Anoplolepis longipes, Sitotroga cerealella, and Culex quinquefasciatus (Gunawardena and Herath 1991). However, so far, there has been no report on the control effect of 2,4-di-tertbutylphenol, n-tridecane, or 3,3-dimethyloctane on $M$. incognita. The discovery of this study may provide a new strategy for controlling $M$. incognita.

This study also found that 3,3-dimethyloctane and n-tridecane were attractive to $M$. incognita at low concentrations $(0.25 \mathrm{mmol} /$ liter). It seems likely that these substances are normally secreted by tomato roots, but only at low levels; as such, they may be used as a signal by RKN searching for a host. Colonization of the root system by $B$. cereus $\mathrm{BCM} 2$ induces the tomato roots to produce larger amounts of these compounds, which at higher concentrations may repel RKN. Endophytes that better ensure the health of their hosts and provide protection from foreign organisms may be more likely to survive; thus, what may be beneficial to the host plant is also beneficial to the endophyte.

Although 2,4-di-tert-butylphenol and 3,3-dimethyloctane appear to be involved in repelling $M$. incognita from the root system, root exudates have a complex chemistry, and other components may be involved in resistance. More research is needed to confirm that 2,4-di-tert-butylphenol and 3,3-dimethyloctane are essential for reducing $M$. incognita infection of tomato roots. Plant root exudates play an important role in plant-nematode interactions. The phytochemicals are considered to be good candidates for biological pesticides, and it should be possible to develop chemically synthesized model compounds that make the plant less attractive to plantparasitic nematodes, as part of an environmentally benign nematicide preparation.

\section{Acknowledgments}

We thank H. Li at the Department of Plant Pathology in Nanjing Agricultural University for kindly providing $M$. incognita egg masses. We also thank Dr. Emily
Drummond at the University of British Columbia for her assistance with English language and grammatical editing of the manuscript.

\section{Literature Cited}

Abad, P., and Gouzy, J. 2008. Genome sequence of the metazoan plant-parasitic nematode Meloidogyne incognita. Nat. Biotechnol. 26:909-915.

Adam, M., Heuer, H., and Hallmann, J. 2014. Bacterial antagonists of fungal pathogens also control root-knot nematodes by induced systemic resistance of tomato plants. PLoS One 9:e90402.

Affokpon, A., and Coyne, D. L. 2011. Biocontrol potential of native Trichoderma isolates against root-knot nematodes in West African vegetable production systems. Soil Biol. Biochem. 43:600-608.

Backman, P. A., and Sikora, R. A. 2008. Endophytes: An emerging tool for biological control. Biol. Control 46:1-3.

Baiocchi, T., Lee, G., Choe, D. H., and Dillman, A. R. 2017. Host seeking parasitic nematodes use specific odors to assess host resources. Sci. Rep. 7:6270.

Bais, H. P., and Weir, T. L. 2006. The role of root exudates in rhizosphere interactions with plants and other organisms. Annu. Rev. Plant Biol. 57: 233-266.

Bao, Q., Ling, L., and Xiao, X. 2011. Comparative tests on different methods for content of soil organic matter. J. Hohai Univ. 39:34-38.

Chitwood, D. J. 2002. Phytochemical based strategies for nematode control. Annu. Rev. Phytopathol. 40:221-249.

Dutta, T. K., and Powers, S. J. 2012. Effect of small lipophilic molecules in tomato and rice root exudates on the behaviour of Meloidogyne incognita and M. graminicola. Nematology 14:309-320.

Fan, J. W., and Hu, C. L. 2014. Jasmonic acid mediates tomato's response to root knot nematodes. J. Plant Growth Regul. 34:196-205.

Gray, N. F. 1984. Ecology of nematophagous fungi: Comparison of the soil sprinkling method with the Baermann funnel technique in the isolation of endoparasites. Soil Biol. Biochem. 16:81-83.

Gunawardena, N. E., and Herath, H. M. W. K. B. 1991. Significance of medium chain $\mathrm{n}$-alkanes as accompanying compounds in hemipteran defensive secretions: An investigation based on the defensive secretion of Coridius janus. J. Chem. Ecol. 17:2449-2458.

Hallmann, J., Mekete, T., Kiewnick, S., and Sikora, R. 2009. Endophytic bacteria from Ethiopian coffee plants and their potential to antagonise Meloidogyne incognita. Nematology 11:117-127.

Hallmann, J., Quadt-Hallmann, A., Rodriguez-Kabana, R., and Kloepper, J. W. 1998. Interactions between Meloidogyne incognita and endophytic bacteria in cotton and cucumber. Soil Biol. Biochem. 30:925-937.

Hashem, M., and Abo-Elyousr, K. A. 2011. Management of the root-knot nematode Meloidogyne incognita on tomato with combinations of different biocontrol organisms. Crop Prot. 30:285-292.

Hu, H.-J., and Chen, Y.-L. 2017. Endophytic Bacillus cereus effectively controls Meloidogyne incognita on tomato plants through rapid rhizosphere occupation and repellent action. Plant Dis. 101:448-455.

Hussey, R. S., and Barker, K. R. 1973. Comparison of methods of collecting inocula of Meloidogyne spp., including a new technique. Plant Dis. Rep. 57:1025-1028.

Jie, W. U., Lin, Z. Z., Guo, Z. J., and Lu, J. X. 2006. Influences of straw mulching treatment on soil physical and chemical properties and crop yields. Southwest China J. Agric. Sci. 19:192-195.

Le, H. T. T., and Padgham, J. L. 2016. Developmental and behavioural effects of the endophytic Fusarium moniliforme Fe14 towards Meloidogyne graminicolain rice. Ann. Appl. Biol. 169:134-143.

Lopes, R., Tsui, S., Goncalves, P., and de Queiroz, M. V. 2018. A look into a multifunctional toolbox: Endophytic Bacillus species provide broad and underexploited benefits for plants. World J. Microbiol. Biotechnol. 34:94.

Niu, Q., and Huang, X. 2010. A Trojan horse mechanism of bacterial pathogenesis against nematodes. Proc. Natl. Acad. Sci. USA 107:16631-16636.

Niu, Q., and Tian, Y. 2011. Overexpression of the key virulence proteases Bace16 and Bae16 in Bacillus nematocida B16 to improve its nematocidal activity. J. Mol. Microbiol. Biotechnol. 21:130-137.

Omacini, M., Semmartin, M., Pérez, L. I., and Gundel, P. E. 2012. Grassendophyte symbiosis: A neglected aboveground interaction with multiple belowground consequences. Appl. Soil Ecol. 61:273-279.

Padgham, J. L., and Sikora, R. A. 2007. Biological control potential and modes of action of Bacillus megaterium against Meloidogyne graminicola on rice. Crop Prot. 26:971-977.

Pradana, A. P., Putri, D., and Munif, A. 2015. Exploration of endophytic bacteria from root of adam hawa plant and their potency as a biocontrol agents and plant growth promoting agents on rice. J. Fitopatol. Indones. 11:73-78.

Sang, M. K., Kim, J. D., Kim, B. S., and Kim, K. D. 2011. Root treatment with rhizobacteria antagonistic to Phytophthora blight affects anthracnose occurrence, ripening, and yield of pepper fruit in the plastic house and field. Phytopathology 101:666-678.

Sang, M. K., and Kim, K. D. 2012. The volatile-producing Flavobacterium johnsoniae strain GSE09 shows biocontrol activity against Phytophthora capsici in pepper. J. Appl. Microbiol. 113:383-398.

Schneider, S. M., and Rosskopf, E. N. 2003. United States Department of Agriculture-Agricultural Research Service research on alternatives to methyl bromide: Pre-plant and post-harvest. Pest Manag. Sci. 59:814-826. 
Sikora, R. A., and Dababat, A. E.-F. 2007. Influence of the mutualistic endophyte Fusarium oxysporum 162 on Meloidogyne incognita attraction and invasion. Nematology 9:771-776.

Sikora, R. A., Schafer, K., and Dababat, A. A. 2007. Modes of action associated with microbially induced in planta suppression of plant-parasitic nematodes. Australas. Plant Pathol. 36:124-134.

Singh, U. B., and Sahu, A. 2013. Can endophytic Arthrobotrys oligospora modulate accumulation of defence related biomolecules and induced systemic resistance in tomato (Lycopersicon esculentum Mill.) against root knot disease caused by Meloidogyne incognita. Appl. Soil Ecol. 63:45-56.

$\mathrm{Su}, \mathrm{L}$., and Shen, Z. 2017. Isolation of antagonistic endophytes from banana roots against Meloidogyne javanica and their effects on soil nematode community. Front. Microbiol. 8:2070.

Tayung, K., Barik, B. P., and Jha, D. K. 2010. Antifungal activity and biocontrol potential of metabolite produced by an endophytic Fusarium (MTCC-9622) against some postharvest pathogens. Int. J. Agric. Technol. 6:409-419.

Van Hecke, M. M., Treonis, A. M., and Kaufman, J. R. 2005. How does the fungal endophyte Neotyphodium coenophialum affect tall fescue (Festuca arundinacea) rhizodeposition and soil microorganisms? Plant Soil 275: 101-109.

Vetrivelkalai, P., Sivakumar, M., and Jonathan, E. I. 2010. Biocontrol potential of endophytic bacteria on Meloidogyne incognita and its effect on plant growth in bhendi. J. Biopesticides 3:452-457.
Vos, C., and Schouteden, N. 2013. Mycorrhiza-induced resistance against the root-knot nematode Meloidogyne incognita involves priming of defense gene responses in tomato. Soil Biol. Biochem. 60:45-54.

Wang, R., Zhou, B., Zhang, F., and Zhang, Q. 2005. Allelopathic effects of root extracts of egg plants on Verticillium wilt (Verticillium dahliae). Allelopathy J. 15:75-83.

Xiong, J., and Zhou, Q. N. 2015. Systemic nematicidal activity and biocontro efficacy of Bacillus firmus against the root-knot nematode Meloidogyne incognita. World J. Microbiol. Biotechnol. 31:661-667.

Yang, G., and Zhou, B. 2016. Effects of tomato root exudates on Meloidogyne incognita. PLoS One 11:e0154675.

Yoon, M.-A., and Tae-Sook, J. 2006. Antioxidant effects of quinoline alkaloids and 2,4-di-tert-butylphenol isolated from Scolopendra subspinipes. Biol. Pharm. Bull. 29:735-739.

$\mathrm{Yu}, \mathrm{Z}$., and Xiong, J. 2015. The diverse nematicidal properties and biocontrol efficacy of Bacillus thuringiensis Cry6A against the root-knot nematode Meloidogyne hapla. J. Invertebr. Pathol. 125:73-80.

Zhang, F., and Peng, D. 2012. In vitro uptake of $140 \mathrm{kDa}$ Bacillus thuringiensis nematicidal crystal proteins by the second stage juvenile of Meloidogyne hapla. PLoS One 7:e38534.

Zhao, L. L., Wei, W., Kang, L., and Sun, J. H. 2007. Chemotaxis of the pinewood nematode, Bursaphelenchus xylophilus, to volatiles associated with host pine, Pinus massoniana, and its vector Monochamus alternatus. J. Chem. Ecol. 33: 1207-1216. 